Research Article

\title{
Deep Learning-Based Magnetic Resonance Imaging in the Evaluation of Tumor, Node, and Metastasis Staging of Renal Cancer
}

\author{
Guocan Han $\mathbb{D}^{1},{ }^{1}$ Weifeng $\operatorname{Lin} \mathbb{D}^{2},{ }^{2}$ and Wei Lin $\mathbb{D}^{1}$ \\ ${ }^{1}$ Department of Radiology, Sir Run Run Shaw Hospital, School of Medicine, Zhejiang University, Hangzhou 310016, \\ Zhejiang, China \\ ${ }^{2}$ Department of Radiology, Prison Central Hospital of Zhejiang Province, Hangzhou 310020, Zhejiang, China
}

Correspondence should be addressed to Wei Lin; linwei7012@zju.edu.cn

Received 27 August 2021; Accepted 3 November 2021; Published 13 December 2021

Academic Editor: Gustavo Ramirez

Copyright (C) 2021 Guocan Han et al. This is an open access article distributed under the Creative Commons Attribution License, which permits unrestricted use, distribution, and reproduction in any medium, provided the original work is properly cited.

This study was aimed to investigate the diagnostic accuracy of magnetic resonance imaging (MRI) based on deep dictionary learning in TNM (tumor, node, and metastasis) staging of renal cell carcinoma. In this study, 82 patients with renal cancer were selected as the research object. The results were diagnosed by deep dictionary learning MRI, and TNM staging was performed by professional imaging personnel. MRI image will be reconstructed after deep dictionary learning to improve its image recognition ability. The pathological diagnosis will be handed over to the physiological pathology laboratory of the hospital for diagnosis. The staging results were compared with the pathological diagnostic staging results, and the results were analyzed by consistency statistics to evaluate the diagnostic value. The results showed that T staging was significantly consistent with the pathological diagnosis. 2 cases were misdiagnosed, and the accuracy rate was $97.56 \%$. Compared with the pathological diagnosis, $\mathrm{N}$ staging had less obvious consistency. 10 cases were misdiagnosed, and the accuracy rate was $87.80 \%$. M staging was significantly consistent with the pathological diagnosis. 4 cases were misdiagnosed. The accuracy rate was $95.12 \%$. After laparotomy, it was found that 37 patients had emboli and 45 patients had no emboli, while 40 patients had emboli and 42 patients had no emboli by MRI. The accuracy rate was $96.34 \%$. The results showed that in the evaluation of TNM staging by MRI imaging based on deep dictionary learning in patients with renal cell carcinoma, the diagnostic results of $\mathrm{N}$ staging and $\mathrm{M}$ staging were highly consistent with the pathological diagnosis, while the diagnostic results of $\mathrm{T}$ staging were slightly less accurate, and the diagnostic consistency was good. The results can provide effective support for the clinical application of MRI imaging based on deep dictionary learning as the clinical diagnosis of TNM staging of renal cell carcinoma.

\section{Introduction}

A renal tumor is a general term for tumor lesions that occur in the kidney that is one of the most common sites of tumors. The renal tumor mostly occurs in renal parenchyma and urinary tubular epithelial system, which is fully known as renal carcinoma (RC) [1]. RC generally originates from different parts of renal proximal convoluted tubules and urinary tubules, but does not include tumors from renal parenchyma and renal pelvis tumors. The exact etiology of renal cell carcinoma is not clear, but it has been confirmed that it can increase the risk with factors such as heredity, obesity, smoking, alcohol, hypertension, and its treatment [2]. Renal cancer often has no obvious clinical symptoms in the early stage. Most patients with renal cancer are asymptomatic renal cancer diagnosed during physical examination, accounting for more than $60 \%$ of the total number of patients with renal cancer. When there are typical symptoms, such as blood in urine and low back pain, and a small number of patients' abdominal masses occurring, the disease often develops. Among them, about $30 \%$ of patients will have paraneoplastic syndrome, with clinical manifestations of abnormal liver function, hypertension, anemia, hypercalcemia, neuromuscular disease, and other 
symptoms, while about $25 \%$ of patients will have bone pain, fracture, hemoptysis, and other symptoms caused by tumor metastasis [3, 4]. According to the World Health Organization (WHO) data released in 2020, RC is the ninth of world's new incidence rate of male cancer, a total of 270 thousand people. The incidence of renal cell carcinoma is dominated by male patients, Western developed countries, and urban population. In China, the high incidence age of renal cancer is about 50 years, the ratio of male to female is $1.83: 1$, and the ratio of urban to rural populations is $4.31: 1$. At present, the number of patients with renal cancer is increasing year by year at an average rate of $6.5 \%$ per year [5-7]. At present, the treatment of renal cell carcinoma is mainly divided into surgical treatment and nonsurgical treatment. The surgical treatment is not only the first choice for the treatment of renal cell carcinoma, but is also recognized as a curable treatment for renal cell carcinoma. The nonsurgical treatment is currently targeted at patients with advanced renal cell carcinoma. Most of them are based on medical treatment, supplemented by a variety of targeted drugs or improved immunotherapy with unilateral nephrectomy [8-10].

The diagnosis of renal cell carcinoma is particularly important because of its early curability. At present, the diagnostic instrument for renal cancer mainly focuses on laboratory examination, imaging examination, and pathological examination. Among them, laboratory tests are generally based on physiological data such as physiological corresponding hormones and cells, which are mainly aiming at the preoperative condition, liver and kidney functions, and prognostic evaluation indexes of patients. The pathological examination is a necessary condition for the diagnosis of renal cell carcinoma, but its operation is complex and difficult to be used as a means of routine examination and diagnosis. The general clinical diagnosis is mainly based on imaging examination $[11,12]$.

Common imaging examinations include chest $\mathrm{X}$-ray film, abdominal ultrasound, abdominal CT (computed tomography), and abdominal magnetic resonance imaging (MRI). The general diagnostic coincidence rate is more than $80 \%$, which has good diagnostic value for the diagnosis of clinical patients with renal cancer $[13,14]$.

TNM (tumor, node, and metastasis) staging system is a common evaluation method for malignant tumor staging. $\mathrm{T}$ refers to tumor, which refers to the increase in tumor volume and the involvement range of adjacent tissues, and is divided into $\mathrm{T} 1 \sim \mathrm{T} 4$. $\mathrm{N}$ refers to lymph node involvement, divided into N0 N3, where N0 refers to lymph node not involved. M refers to metastasis, which refers to distant metastasis, which is divided into nonmetastatic M0 and metastatic M1. The staging system can assist doctors in the diagnosis and treatment of patients' tumors and is widely used in clinic. The judgment basis of staging comes from the imaging diagnosis of patients [15].

In recent years, computer-aided diagnosis (CAD) has been widely used in the medical field. The medical influence is analyzed and calculated by computer to improve the image accuracy, which greatly improves the diagnostic efficiency and accuracy. The progress of artificial intelligence makes the technology have a new development direction, especially the combination of deep dictionary learning and MRI image, which greatly improves the play of MRI image in exploring lesions and pathological judgment. Therefore, the combination of deep dictionary learning and MRI will have a good application prospect in the field of renal cancer diagnosis [16-19].

In summary, this study will improve the ability of clinical diagnosis through the combination of deep dictionary learning and magnetic resonance, so as to avoid the problems of misdiagnosis and low accuracy of CT and B ultrasound. 82 patients with renal cancer in the hospital were selected as the research object, and the patients were diagnosed by MRI with deep dictionary learning. The clinical value of TNM staging in renal cell carcinoma was judged by comparing it with the pathological diagnosis, to provide a more safe, effective, and convenient diagnostic method for the staging diagnosis of renal cancer patients, verify its effectiveness, and carry out clinical promotion.

\section{Materials and Methods}

2.1. Research Object. Eighty-two patients with renal cancer in the hospital were collected as research objects, including 64 males and 18 females, aged from 27 to 83 years, with an average age of $(54.73 \pm 4.59)$ years. The main clinical manifestations were abdominal mass in 35 cases, abdominal pain in 23 cases, hematuria in 11 cases, fever in 7 cases, and no obvious symptoms in 6 cases. This study has been approved by the Medical Ethics Committee of the hospital. Patients and their families have been informed of this study and signed the informed consent.

Inclusion criteria: (1) patients with confirmed renal cancer [20]

Exclusion criteria: (1) patients with other serious heart, kidney, liver, and other organ diseases; (2) patients with severe mental illness and unable to communicate normally; (3) family members and patients are reluctant to participate

2.2. Treatment Options and Course of Treatment. All cases meeting the access requirements were performed with deep dictionary learning magnetic resonance, and the instrument was an MRI system. The patients were bedridden, and plain scan and dynamic enhanced scan were performed by a scanner. The plain scan was the transverse section (TRA) axis, including the fat sequence made by T2-weighted image (T2WI), the fat sequence without inhibition, and the diffusion-weighted imaging (DWI) sequence. The T2WI was repeated, and the echo time was $800 \mathrm{~ms}$ and $60 \mathrm{~ms}$. The layer thickness was $5-7 \mathrm{~mm}$, and the layer spacing was $1.5-2.5 \mathrm{~mm}$. The contrast agent used Gd-DTPA, $15 \mathrm{~mL}$, through the elbow vein group injection, $2 \mathrm{~mL} / \mathrm{s}$. Imaging was performed within $30 \mathrm{~s}, 50 \mathrm{~s}, 100 \mathrm{~s}$, and $150 \mathrm{~s}$ after injection.

2.3. MRI Image Based on Deep Dictionary Learning. As for deep dictionary learning, this study will choose image reconstruction based on deep dictionary learning to process 
MRI images. Image reconstruction based on deep dictionary learning can reconstruct the image and greatly improve the accuracy of the image for key parts and the embodiment of small tissue parts.

The image reconstruction of deep dictionary learning proposed in this study mainly includes two steps: training dictionary and image reconstruction. The dictionary learning algorithm is used to deeply learn the preselected sample data, and finally, a dictionary $D$ containing the characteristics of the sample data is obtained. The image reconstruction is to decompose the sample image into several image blocks and solve the coefficient $Z$ corresponding to the image block according to the trained dictionary $E$. Finally, the dictionary $E$ and the solved coefficient $Z$ are reconstructed according to the image block to obtain the reconstructed test image. The coefficient $\mathrm{Z}$ of the image block can be solved according to

$$
\min _{z}\left\|X_{i}-E Z_{i}\right\|_{2}^{2}+\left\|Z_{i}\right\|_{1}
$$

where $X_{i}$ represents the image block coefficient, and the dictionary $E$ obtained through training and the obtained coefficient $Z_{i}$ can reconstruct the image block $Y_{i}$ :

$$
Y_{i}=E \cdot Z_{i}
$$

The reconstructed image $Y$ can be obtained by combining all the image blocks $Y_{i}$.

Dictionary updates are also conducted layer by layer, and the equation is as follows:

$$
E=X \cdot Z^{-1}
$$

The coefficient update is the least-squares solution of matrix $X_{i}$ constructed by merging the product of the input data of each layer and the current corresponding coefficient according to the row by equation (3) and the matrix $A_{i}$ constructed by merging the same unit diagonal matrix according to the row on the basis of the updated dictionary of each layer. The solution will be used as the coefficient matrix $Z_{i}$ and satisfies

$$
\begin{gathered}
\min _{z}\left\|X-E_{n-1} Z\right\|_{F}^{2} \longrightarrow Z_{n}, \\
\min _{E}\left\|X-E Z_{n}\right\|_{F}^{2} \longrightarrow E_{n} .
\end{gathered}
$$

In this experiment, the image data obtained by MRI scanning are processed as follows: the collected scanning image $(500 \times 512)$ is divided into $128 \times 2$ image blocks as the sample data of dictionary training. The dimension of the training sample is $256 \times 1120$. The algorithm iteration $N=100$ and the dictionary are three-tier structure. The first 1,000 columns in the training sample are selected and initialized as dictionary $E 1$, and $E 1$ is $256 \times 1000$. The obtained $E 1$ is normalized, and a new $E 1$ is obtained. The inverse of the new $E 1$ matrix and the inner product of $X$ are calculated, and then, the coefficient matrix $Z 1$ of the first layer of dictionary learning is obtained, which is $1000 \times 1120$.

The obtained coefficient matrix is input into the second layer dictionary learning. The first 800 columns of $Z 1$ are selected to initialize E2, and the $E 2$ is also processed. The coefficient matrix $Z 2$ of the second layer dictionary learning is $800 \times 1120$.

By the same operation, the coefficient matrix $Z 3$ of the third layer dictionary learning is obtained, which is $600 \times 1120$.

The algorithm iterative is set as 100 times. When the number of iterations is insufficient, the dictionary can be updated layer by layer according to equation (3). Fixation dictionary and matrix $X_{i}, A_{i}(i=1,2,3)$ are built in each layer. According to equation (4), the least-squares solution about $X_{i}, A_{i}$ is obtained to update the corresponding coefficient. All updates are updated layer by layer using alternating minimization.

The update is stopped when the error of each layer update is less than the threshold. Because all decompositions are linear during dictionary learning, the final dictionary can be represented as follows:

$$
E=E_{1} \cdot E_{2} \cdot E_{3} .
$$

The specific flowchart of the algorithm is shown in Figure 1.

2.4. Simulation Experiment. To evaluate the effect of image reconstruction, the commonly used indexes are to calculate the mean square error (MSE) and peak signal-tonoise ratio (PSNR) of reconstructed image and test image. The calculation equation of mean square error is as follows:

$$
\text { mean }- \text { square error }=\frac{1}{L \times W} \sum_{i=1}^{L} \sum_{j=1}^{W}\left(C_{1}(i, j)-C_{2}(i, j)\right)^{2},
$$

where $C_{1}$ represents the reconstructed image, $C_{2}$ is the test image, $L$ is the image length, and $W$ is the image width. The smaller the MSE, the finer the reconstructed image.

PSNR is the most commonly used evaluation index in the field of image processing, and its equation is as follows:

$$
\text { peak signal }- \text { to }- \text { noise ratio }=10 \log _{10}\left(\frac{\left(2^{b}-1\right)^{2}}{M S E}\right),
$$

where $b$ represents the number of bits per pixel. The greater the PSNR, the higher the quality of image reconstruction.

The data samples are selected and divided into $8 \times 8$, 10,000 images are selected as training samples, and the depth dictionary learning model training data are constructed to obtain the deep-seated features of the image. KSVD [21, 22] algorithm and stacked autoencoder (SAE) [23, 24] are selected for data training and image reconstruction, which is compared with the reconstructed image of depth dictionary learning model.

The experimental environment is Win10 system, CPU: Intel (R) Celeron (R) CPU 1007U @ 1.50 GHz, and MATLAB platform. 


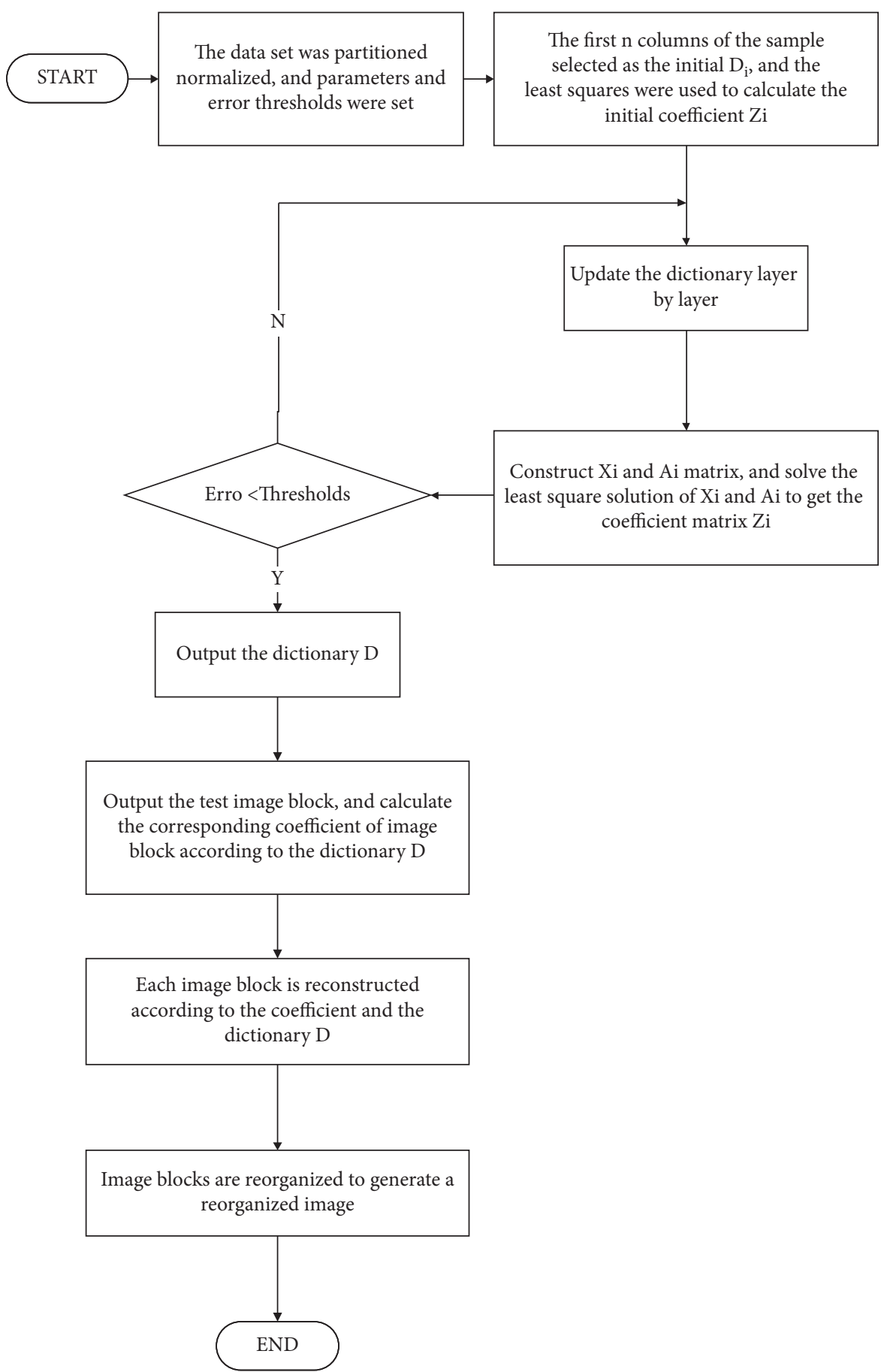

FIgURE 1: Image flow diagram based on deep dictionary learning.

2.5. TNM Staging. The specific stage control standard of TNM refers to the newly revised TNM stage standard in 1997 [25]. Two professional diagnostic physicians made an independent judgment on the basis of understanding the patient's clinical symptoms and medical history, and not knowing the final diagnostic results. The final conclusion was reached after discussion on the cases with different diagnostic results.
2.6. Statistical Study. In this study, the MedCalc version is used for data processing as analysis and statistics software, the kappa consistency test is used for correlation, and the test level is $\propto=0.05$. The reference evaluation of $K$ (kappa) value is as follows: $K \leq 0.40$, poor diagnostic consistency; $0.40<K \leq 0.75$, good diagnostic consistency; and $0.75<K \leq 1$, excellent diagnostic consistency. 


\section{Results}

3.1. Algorithm Simulation Results. Figure 2 shows the input test sample. The test chart is divided into image blocks of $8 \times 8$. The reconstruction results of the four algorithm models are shown in Figure 3.

Figure 3(a) is SAE-reconstructed image. Figure 3(b) is the reconstructed image of single-layer dictionary learning. Figure 3(c) is the reconstructed image of KSVD. Figure 3(d) is the dictionary reconstruction image after the deep dictionary learning model training. The corresponding PSNR and MSE are compared as shown in Figure 4. By comparing the reconstructed image and the reconstructed image with the original PSNR and MSE, the image reconstruction effect under the deep dictionary learning model is significantly better than SAE reconstruction, single-layer dictionary reconstruction model, and KAVD reconstruction. Experiments show that deep dictionary learning has a good performance in image reconstruction.

\subsection{Comparison Results of TNM in Patients with Renal Cell} Carcinoma. After comparative evaluation, the results of $\mathrm{T}$ stage diagnosis under MRI based on deep dictionary learning and pathological diagnosis in TNM staging of patients with renal cancer are shown in Figure 5. The accuracy of MRI in the diagnosis of T stage was $97.56 \%$, and 2 cases were misdiagnosed. The diagnosis of T3 stage was consistent, 1 case of T2 stage was misdiagnosed as T1 stage, and 1 case was misdiagnosed as T4 stage. After calculation, $K=0.822$.

After comparative evaluation, the results of $\mathrm{N}$ stage diagnosis under MRI based on deep dictionary learning and pathological diagnosis in TNM staging of patients with renal cancer are shown in Figure 6. The accuracy of MRI in the diagnosis of $\mathrm{N}$ stage was $87.80 \%$, and 10 cases were misdiagnosed. Among them, 5 cases of N2 stage were misdiagnosed as $\mathrm{N} 1$ stage, and 5 cases of $\mathrm{N} 2$ stage were misdiagnosed as N1 stage. After calculation, $K=0.735$.

After comparison and evaluation, the results of $M$ stage diagnosis under MRI based on deep dictionary learning and pathological diagnosis of patients with renal cancer in TNM staging are shown in Figure 7. The accuracy of MRI in the diagnosis of $\mathrm{M}$ stage was $95.12 \%$, and 4 cases were misdiagnosed. Four patients with M1 stage were misdiagnosed as M0 stage. After calculation, $K=0.802$.

After comparative evaluation, the results of diagnosis under MRI based on deep dictionary learning and pathological diagnosis of renal vein thrombus carcinoma in patients with renal cancer are shown in Figure 8. The accuracy of MRI in diagnosing embolus was $96.34 \%$, and 3 cases were misdiagnosed.

\section{Discussion}

TNM staging was first proposed by Pierre Denoix of France from 1943 to 1952, and then, the international staging standard was jointly established by the American Joint Committee on Cancer and the Union for International

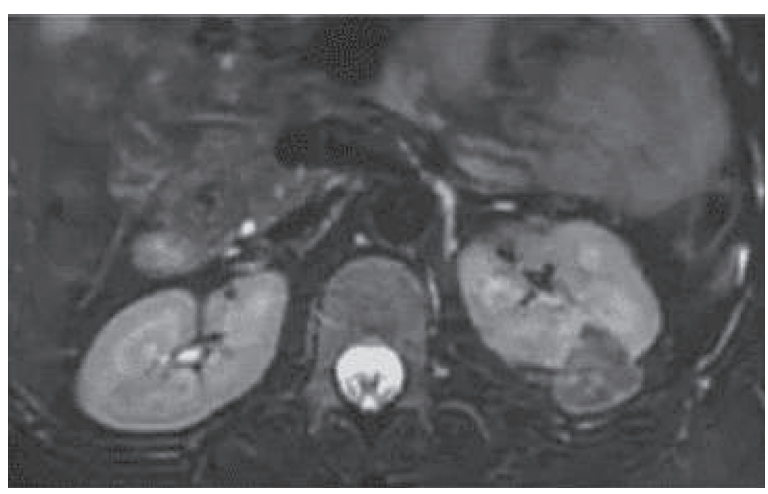

Figure 2: Test chart.

Cancer Control. The first edition of TNM Classification of Malignant Tumors was published in 1968. At present, TNM staging has become a standard method for clinical staging of malignant tumors. In this study, patients were diagnosed by TNM staging through MRI imaging and pathological examination of deep dictionary learning, so as to determine whether MRI imaging based on deep dictionary learning can be popularized as a means of clinical diagnosis.

Firstly, the verification experiment of deep dictionary learning MRI is carried out. In this study, deep dictionary learning is used to reconstruct the magnetic resonance image to improve the accuracy of the image. Through the verification experiment, it is confirmed that the depth dictionary learning has a better improvement in image accuracy compared with other image reconstruction methods.

In this study, 82 patients in the hospital were selected as research objects, and deep learning-based MRI was performed on all of them, and professional imaging physicians were invited for diagnosis. In the final results, two cases were misdiagnosed at $\mathrm{T}$ stage, and the accuracy rate was $97.56 \% .10$ cases were misdiagnosed with $\mathrm{N}$ stage, and the accuracy was $87.8 \%$. 4 cases were misdiagnosed in M stage, and the accuracy was $95.12 \%$. 3 cases of renal venous thrombus carcinoma were misdiagnosed, and the accuracy rate was $96.34 \%$. In this study, the accuracy of T stage, $M$ stage, and renal venous thromboembolic carcinoma was high, and only $\mathrm{N}$ stage had more error, but it was still within the range of good consistency diagnosis. The cause of misdiagnosis was preliminarily speculated to be that there was little difference between lymph node metastasis and lymphoproliferative imaging in MRI, which was easy to be confused in $\mathrm{N}$ stage diagnosis. It was difficult to distinguish benign tumor from malignant tumor, leading to diagnostic error.

In this study, it is determined that MRI based on deep dictionary learning can effectively show the tumor size, tumor location, tumor invasion range, tumor surrounding tissue involvement range, tumor stage, tumor embolus, and other structures and parts in the clinical diagnosis of renal cell carcinoma, which is convenient for doctors' clinical diagnosis. In addition, because of its clear presentation of the lesion tissue, it can also be used as an imaging guidance scheme for surgery and prognosis recovery comparison and secondary surgery. 


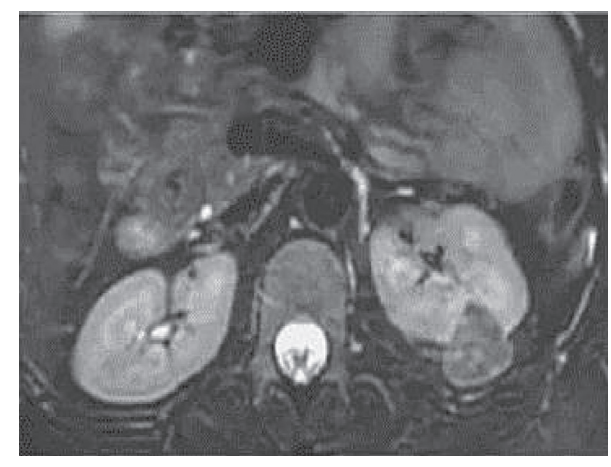

(a)

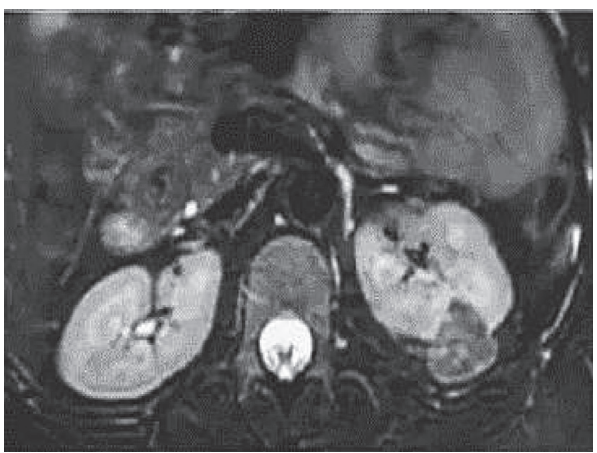

(c)

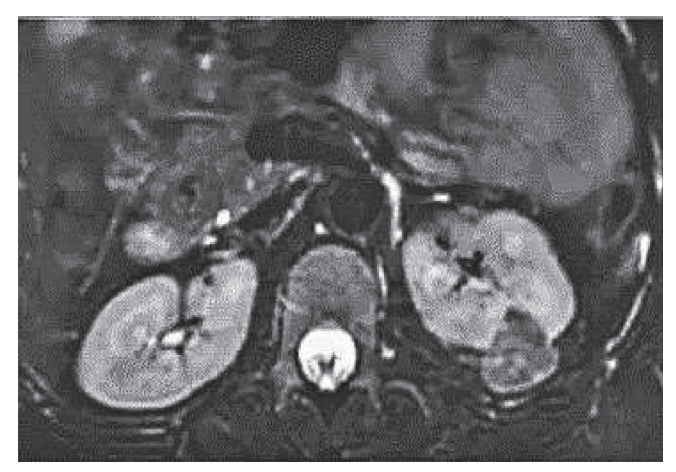

(b)

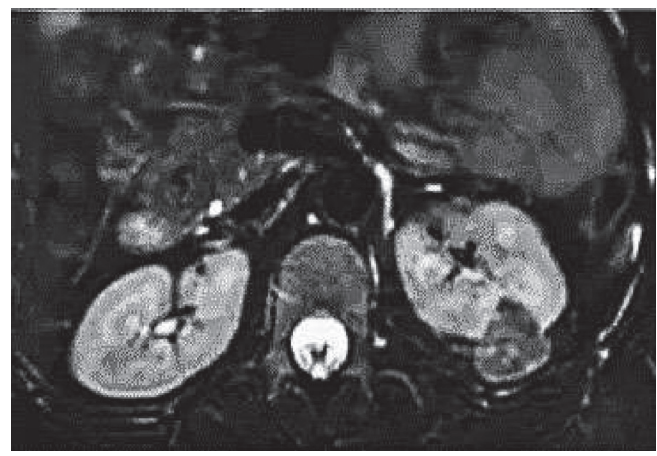

(d)

FIGURE 3: Reconstructed image results. (a) SAE reconstitution. (b) Single-layer dictionary reconstruction. (c) KAVD reconstitution. (d) Deep dictionary reconstruction.

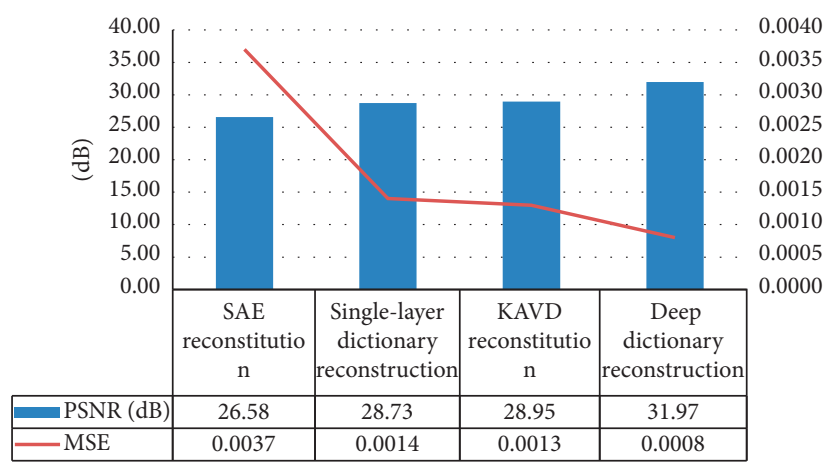

FIGURE 4: Comparison of simulation experimental results of four reconstruction methods.

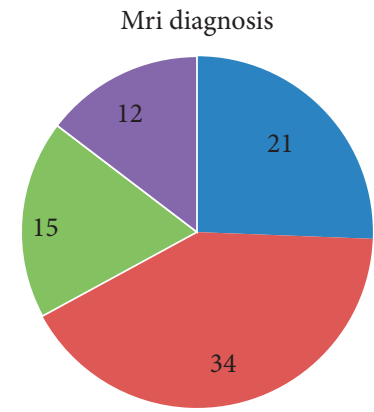

$$
\begin{array}{r}
\mathrm{T} 1 \\
\mathrm{~T} 2
\end{array}
$$

T3

(a)

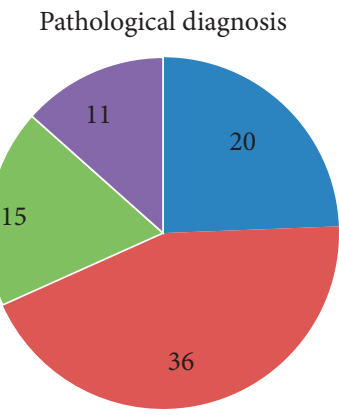

$\begin{array}{ll}\mathrm{T} 1 & \mathrm{~T} 3 \\ \mathrm{~T} 2 & \mathrm{~T} 4\end{array}$

(b)

FIgURE 5: Comparison of T stage diagnostic results. (a) MRI diagnosis and (b) pathological diagnosis. 

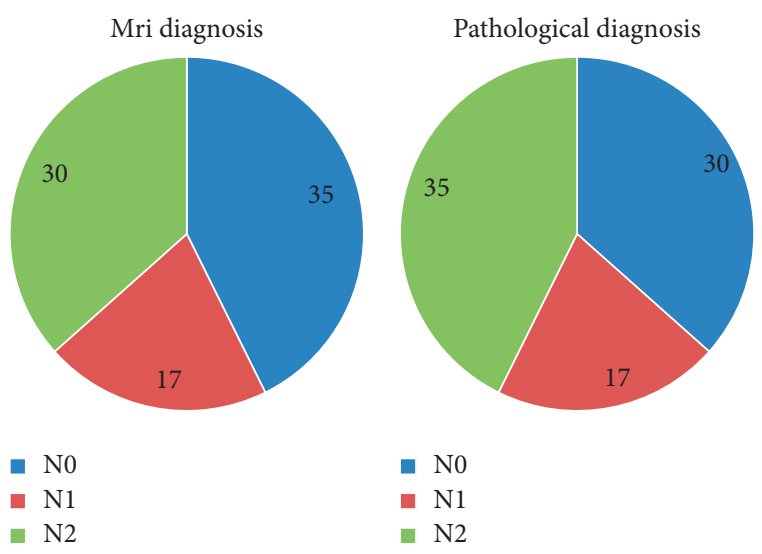

(a)

(b)

FIgURE 6: Comparison of $\mathrm{N}$ stage diagnostic results. (a) MRI diagnostic results and (b) pathological diagnostic results.

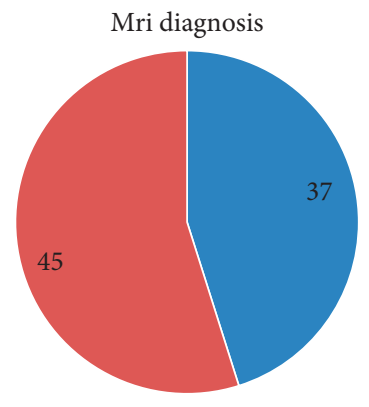

- M0

- M1

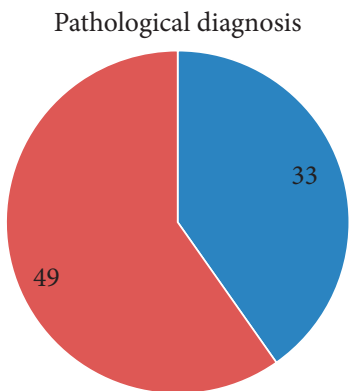

- M0

- M1

(b)
FIgURE 7: Comparison of $M$ stage diagnostic results. (a) MRI diagnostic results and (b) pathological diagnostic results.

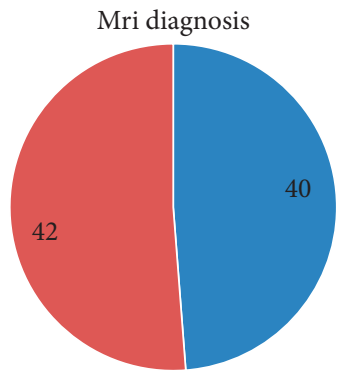

- embolus

- No embolus

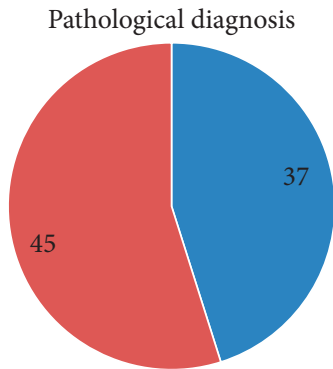

- embolus

- No embolus (a)

(b)

Figure 8: Comparison of embolic diagnostic results. (a) MRI diagnostic results and (b) pathological diagnostic results.

Zhao et al. [26] diagnosed stage I and stage II renal cell carcinoma by MRI under in-depth learning of 376 cases of 430 renal cell carcinomas. The results showed that MRI had high accuracy in the diagnosis, prediction, and histological grading of stage I and stage II renal cell carcinoma. Xi et al. [27] experimented with the accuracy of conventional MRI in the diagnosis of benign and malignant lesions of renal cell carcinoma. The results showed that MRI based on deep dictionary learning has considerable accuracy, sensitivity, and specificity in the diagnosis of benign and malignant lesions of renal cell carcinoma.

\section{Conclusion}

In this study, 82 patients with renal tumors were selected as the research object, and their TNM staging was evaluated by MRI based on deep dictionary learning and compared with the results of pathological examination. The results showed that the TNM staging results of MRI based on deep dictionary learning were in good agreement with the pathological examination. In particular, for the judgment of $\mathrm{T}$ stage and $\mathrm{M}$ stage, there is excellent consistency. It is proved that this detection method can be used in the clinical diagnosis of renal cell carcinoma and is worthy of popularization. In this experiment, there are few diagnostic items in selected patients, and only TNM staging and renal vein thrombosis cancer are judged. More diagnostic items can be compared in the future to further determine the role of MRI based on deep dictionary learning in the diagnosis of renal cell carcinoma. This study provides clinical data, and for the diagnosis of renal cell carcinoma, it provides a certain development direction.

\section{Data Availability}

The data used to support the findings of this study are available from the corresponding author upon request.

\section{Conflicts of Interest}

The authors declare no conflicts of interest.

\section{Authors' Contributions}

Guocan Han and Weifeng Lin contributed equally to this work.

\section{References}

[1] R. E. Gray and G. T. Harris, "Renal cell carcinoma: diagnosis and management," American Family Physician, vol. 99, no. 3, pp. 179-184, 2019 Feb 1.

[2] N. Petejova and A. Martinek, "Renal cell carcinoma: review of etiology, pathophysiology and risk factors," Biomedical $\mathrm{Pa}$ pers, vol. 160, no. 2, pp. 183-194, 2016 Jun.

[3] M. A. Perazella, R. Dreicer, and M. H. Rosner, "Renal cell carcinoma for the nephrologist," Kidney International, vol. 94, no. 3, pp. 471-483, 2018 Sep.

[4] R. Shingarev and E. A. Jaimes, "Renal cell carcinoma: new insights and challenges for a clinician scientist," American Journal of Physiology - Renal Physiology, vol. 313, no. 2, pp. F145-F154, 2017 Aug 1. 
[5] U. Capitanio, K. Bensalah, A. Bex et al., "Epidemiology of renal cell carcinoma," European Urology, vol. 75, no. 1, pp. 74-84, 2019 Jan.

[6] K. Yoshimura and H. Uemura, "Pharmacotherapies for renal cell carcinoma in Japan," International Journal of Urology, vol. 23, no. 3, pp. 194-202, 2016 Mar.

[7] S. Zhang, B. Wang, F. Zhang, J. Ye, L. Ge, and L. Ma, "Genomic alterations of renal cell carcinoma and clinical implications in the Chinese population," Medical Science Monitor, vol. 25, pp. 2959-2965, 2019 Apr 22.

[8] F. Erlmeier, "Chromophobes Nierenzellkarzinom - diagnostik und Prognostik," Pathologe, Der, vol. 40, no. S3, pp. 252-258, 2019 Dec.

[9] B. Escudier, C. Porta, M. Schmidinger et al., "Renal cell carcinoma: ESMO Clinical Practice Guidelines for diagnosis, treatment and follow-up," Annals of Oncology, vol. 27, no. suppl 5, pp. v58-v68, 2016 Sep.

[10] P. Wiechno, J. Kucharz, M. Sadowska et al., "Contemporary treatment of metastatic renal cell carcinoma," Medical Oncology, vol. 35, no. 12, p. 156, 2018 Oct 27.

[11] G. M. Israel and M. A. Bosniak, "Renal imaging for diagnosis and staging of renal cell carcinoma," Urologic Clinics of North America, vol. 30, no. 3, pp. 499-514, 2003 Aug.

[12] Z. Wang and Y. S. Zhang, "[Metabonome in early diagnosis of renal cell carcinoma]," Zhongguo Yi Xue Ke Xue Yuan Xue Bao, vol. 41, no. 5, pp. 709-713, 2019 Oct 30, Chinese.

[13] H. Zhu, S. Zhao, C. Zuo, and F. Ren, "FDG PET/CT and CT findings of renal cell carcinoma with sarcomatoid differentiation," American Journal of Roentgenology, vol. 215, no. 3, pp. 645-651, 2020 Sep.

[14] S. Ursprung, L. Beer, A. Bruining et al., "Radiomics of computed tomography and magnetic resonance imaging in renal cell carcinoma-a systematic review and meta-analysis," European Radiology, vol. 30, no. 6, pp. 3558-3566, 2020 Jun.

[15] U. Swami, R. H. Nussenzveig, B. Haaland, and N. Agarwal, "Revisiting AJCC TNM staging for renal cell carcinoma: quest for improvement," Annals of Translational Medicine, vol. 7, no. S1, p. S18, 2019 Mar.

[16] G. Liberman and B. A. Poser, "Minimal linear networks for magnetic resonance image reconstruction," Scientific Reports, vol. 9, no. 1, p. 19527, 2019 Dec 20.

[17] S. Albarqouni, U. Konrad, L. Wang, N. Navab, and S. Demirci, "Single-view X-ray depth recovery: toward a novel concept for image-guided interventions," International Journal of Computer Assisted Radiology and Surgery, vol. 11, no. 6, pp. 873-880, 2016 Jun.

[18] Z. Akkus, A. Galimzianova, A. Hoogi, D. L. Rubin, and B. J. Erickson, "Deep learning for brain MRI segmentation: state of the art and future directions," Journal of Digital Imaging, vol. 30, no. 4, pp. 449-459, 2017 Aug.

[19] T. Higaki, Y. Nakamura, F. Tatsugami, T. Nakaura, and K. Awai, "Improvement of image quality at CT and MRI using deep learning," Japanese Journal of Radiology, vol. 37, no. 1, pp. 73-80, 2019 Jan.

[20] R. J. Motzer, G. B. Bolger, B. Boston et al., "Kidney cancer. Clinical practice guidelines in oncology," Journal of the National Comprehensive Cancer Network: Journal of the National Comprehensive Cancer Network, vol. 4, no. 10, pp. 1072-1081, 2006 Nov.

[21] N. Leal, E. Zurek, and E. Leal, "Non-local SVD denoising of MRI based on sparse representations," Sensors, vol. 20, no. 5, p. 1536, 2020 Mar 10.

[22] Z. Chen, J. Zhang, and K. K. Pang, "Adaptive keyhole methods for dynamic magnetic resonance image reconstruction,"
Computerized Medical Imaging and Graphics, vol. 31, no. 6, pp. 458-468, 2007 Sep.

[23] H.-I. Suk, S. W. Lee, S.-W. Lee, and D. Shen, "Latent feature representation with stacked auto-encoder for $\mathrm{AD} / \mathrm{MCI}$ diagnosis," Brain Structure and Function, vol. 220, no. 2, pp. 841-859, 2015 Mar.

[24] Y. Guo, Y. Gao, and D. Shen, "Deformable MR prostate segmentation via deep feature learning and sparse patch matching," IEEE Transactions on Medical Imaging, vol. 35, no. 4, pp. 1077-1089, 2016 Apr.

[25] V. Ficarra, A. Galfano, M. Mancini, G. Martignoni, and W. Artibani, "TNM staging system for renal-cell carcinoma: current status and future perspectives," The Lancet Oncology, vol. 8, no. 6, pp. 554-558, 2007 Jun.

[26] Y. Zhao, M. Chang, R. Wang et al., "Deep learning based on MRI for differentiation of low- and high-grade in low-stage renal cell carcinoma," Journal of Magnetic Resonance Imaging, vol. 52, no. 5, pp. 1542-1549, 2020 Nov.

[27] I. L. Xi, Y. Zhao, R. Wang et al., "Deep learning to distinguish benign from malignant renal lesions based on routine MR imaging," Clinical Cancer Research, vol. 26, no. 8, pp. 1944-1952, 2020 Apr 15. 